\title{
The discriminative power of the ReproQ: a client experience questionnaire in maternity care
}

\author{
Marisja Scheerhagen $^{1,2}$, Henk F van Stel ${ }^{3}$, Arie Franx ${ }^{2}$, Erwin Birnie ${ }^{\text {Corresp., } 4}$, Gouke J Bonsel $^{5}$ \\ 1 Department of Obstetrics and Gynecology, Erasmus Medical Centre, Rotterdam, The Netherlands \\ 2 Department of Obstetrics and Gynecology, University Medical Center Utrecht, Utrecht, The Netherlands \\ 3 Department of Healthcare Innovation and Evaluation, University Medical Center Utrecht, Utrecht, The Netherlands \\ 4 Erasmus School of Health Policy \& Management, Department of Health Technology Assessment, Erasmus University Rotterdam, Rotterdam, The \\ Netherlands \\ 5 Department of Obstetrics and Gynecology, Academic Collaborative Maternity Care, University Medical Center Utrecht, Utrecht, The Netherlands \\ Corresponding Author: Erwin Birnie \\ Email address: birnie@eshpm.eur.nl
}

Background. Aim of the ReproQuestionnaire (ReproQ) is to measure the client's experience with maternity care, following WHO's responsiveness model. To support quality improvement, ReproQ should be able to discriminate best from worst organisational units. Methods. We sent questionnaires to 27,487 third-trimester pregnant women (response $31 \%$ ) and to 37,230 women 6 weeks after childbirth (response 39\%). For analysis we first summarized the ReproQ domain scores into three summary scores: total score (all eight domains), personal score (four personal domains), and setting score (four setting domains). Second, we estimated the proportion of variance across perinatal units attributable to the 'actual' difference across perinatal units using intraclass correlation coefficients (ICCS). Third, we assessed the ability of ReproQ to discriminate between perinatal units based on both a statistical approach using multilevel regression analyses, and a relevance approach based on the minimally important difference (MID). Finally, we compared the domain scores of the best and underperforming units. Results. ICCs ranged between 0.004 and 0.025 for the summary scores, and between 0.002 and 0.125 for the individual domains. ReproQ was able to identify the best and worst performing units with both the statistical and relevance approach. The statistical approach was able to identify four underperforming units during childbirth (total score), while the relevance approach identified 10 underperforming units. Conclusions. ReproQ, a valid and efficient measure of client experiences in maternity care, has the ability to discriminate well across perinatal units, and is suitable for benchmarking under routine conditions. 


\section{The discriminative power of the ReproQ: a client}

\section{2 experience questionnaire in maternity care}

4 Marisja Scheerhagen, $\mathrm{MSc}^{1,2}$, Henk van Stel, $\mathrm{PhD}^{3 \dagger}$, Arie Franx, $\mathrm{PhD}, \mathrm{MD}^{2}$, Erwin

$5 \quad$ Birnie, $\mathrm{PhD}^{4}$, Gouke Bonsel, $\mathrm{PhD}, \mathrm{MD}^{5}$

$7{ }^{1}$ Department of Obstetrics and Gynecology, Erasmus Medical Centre, Rotterdam, The 8 Netherlands

2 Department of Obstetrics and Gynecology, University Medical Center Utrecht, Utrecht,

10 The Netherlands

3 Department of Healthcare Innovation and Evaluation, University Medical Center

12 Utrecht, Utrecht, The Netherlands

${ }^{4}$ Erasmus School of Health Policy \& Management, Department of Health Technology Assessment, Erasmus University Rotterdam, Rotterdam, The Netherlands

${ }^{5}$ Department of Obstetrics and Gynecology, Academic Collaborative Maternity Care,

16 University Medical Center Utrecht, Utrecht, The Netherlands

Corresponding author:

Erwin Birnie PhD 4

Erasmus university

school of health policy and management

Boyle-Building

PO box 1738

3000 DR Rotterdam 
25 The Netherlands.

\section{Abstract}

Background. Aim of the ReproQuestionnaire (ReproQ) is to measure the client's experience with maternity care, following WHO's responsiveness model. To support quality improvement, ReproQ should be able to discriminate best from worst organisational units.

Methods. We sent questionnaires to 27,487 third-trimester pregnant women (response $31 \%$ ) and to 37,230 women 6 weeks after childbirth (response $39 \%$ ). For analysis we first summarized the ReproQ domain scores into three summary scores: total score (all eight domains), personal score (four personal domains), and setting score (four setting domains). Second, we estimated the proportion of variance across perinatal units attributable to the 'actual' difference across perinatal units using intraclass correlation coefficients (ICCs). Third, we assessed the ability of ReproQ to discriminate between perinatal units based on both a statistical approach using multilevel regression analyses, and a relevance approach based on the minimally important difference (MID).

42 Finally, we compared the domain scores of the best and underperforming units. 
43 Results. ICCs ranged between 0.004 and 0.025 for the summary scores, and between

440.002 and 0.125 for the individual domains. ReproQ was able to identify the best and

45 worst performing units with both the statistical and relevance approach. The statistical

46 approach was able to identify four underperforming units during childbirth (total score),

47 while the relevance approach identified 10 underperforming units.

48 Conclusions. ReproQ, a valid and efficient measure of client experiences in maternity

49 care, has the ability to discriminate well across perinatal units, and is suitable for

50 benchmarking under routine conditions. 


\section{Introduction}

53 The performance of health care systems is primarily judged by health outcomes, such

54 as mortality, morbidity, health status, or burden of disease. System performance differs

55 across and within countries, partly caused by differences in the provision of care

56 (Mohangoo et al. 2011; Zeitlin et al. 2013a; Zeitlin et al. 2013b). To highlight the role of

57 provision of care in health system performance, the World Health Organization (WHO)

58 introduced the measurement of client experiences with service provision and service

quality as a cornerstone in health care provider evaluations (Valentine et al. 2007;

60 Valentine et al. 2003).

62 Client experiences with care provision matter for at least two reasons. First, these may guide the client's choice of health care provider, particularly when the health outcomes across providers are about similar (Valentine et al. 2003). Second, better client experiences may contribute to improved health outcomes (Campbell et al. 2000;

Valentine et al. 2007; Valentine et al. 2003). For example, clients who understand their caregiver's explanations are more likely to comply to treatment or lifestyle changes.

To cover a broad spectrum of client experiences, independent from specific system 
71 the so-called Responsiveness concept. Responsiveness is defined as the way a client

72 is treated by the professional and the environment in which the client is treated during a

73 health care encounter (De Silva 2000; Gostin 2002; Valentine et al. 2003). It is based on

74 actual performance in health practice, rather than on organisational features with

75 claimed benefit.

77 Responsiveness data can be used in a universal two stage quality cycle. In the first stage, through aggregated client scores, health care providers are ranked in terms of performance. In the second stage, each underperforming organisation digs into the differences responsible for the deviant result by disaggregation of summary scores into domain scores and/or item scores. The subsequent implementation of improvement measures to target the identified weak points in service delivery should result in measurable improvement, even among average performers (Collins-Fulea et al. 2005). This check of improvement closes the quality cycle.

In maternity care, routine measurement of client experiences is common practice in

87 several countries. However, to our knowledge, a fully implemented two-stage quality cycle is not yet operational in maternity care. (Dzakpasu et al. 2008; Hay 2010; 
90 challenge in performance measurement in maternity care, with clients being

91 predominantly healthy young women, is the discriminative power of a measure to

92 quantify client experiences. Poor outcomes are infrequent, and poor performance of

93 specific client groups or units can easily be compensated by good performance of other

94 client groups or units. Moreover, systematic variation in performance scores across

95 units can originate from systematic variation in performance scores at the unit level

even when clients are comparable across units, or from actual variation in individual

performance scores of clients within units. This so-called nested or hierarchical

structure of performance (units and clients within units) requires a specific statistical

approach to expose the actual performance at the perinatal unit level, but above all a

measure with excellent measurement properties without becoming too lengthy or complex.

The study aim was to evaluate the discriminative power of ReproQ at the perinatal unit

level (a hospital with its associated community midwife practices). ReproQ is a validated

questionnaire to measure client experiences with maternity care based on WHO's

Responsiveness concept (Scheerhagen et al. 2015b). We use two approaches to determine discriminative ability. The first, conventional, approach is to identify poor 
109 average performance score of all perinatal units, taking the nested nature of the data

110 into account. In the second approach, we identified a perinatal unit as poor performer if

111 its aggregated score deviated from the aggregated score of the best performing units by

112 at least a minimally important or 'meaningful' difference (MID). Once discriminatory

113 power is determined, we explore the potential for targeting the areas that need

114 improvement. We hypothesized that ReproQ has sufficient discriminatory power for

115 national implementation in the Dutch maternity care system, if ReproQ shows sufficient

116 discriminatory power in both approaches, and is able to identify targets for

117 improvement. 


\section{Materials \& Methods}

120

121

122

123

124

125

126

127

128

129

ReproQuestionnaire

ReproQ (33 items) consists of two complementary versions; the antepartum

questionnaire addresses women's experiences in the first and second half of

pregnancy, while the postpartum questionnaire addresses women's experiences the childbirth and the subsequent postpartum week.

The eight-domain WHO Responsiveness concept was used as the conceptual base (Valentine et al. 2007; Valentine et al. 2003). The four domains on personal interactions between the client and health professional are: dignity, autonomy, confidentiality and communication. The four domains regarding experiences with the organizational setting are: prompt attention, access to family and community support, quality of basic amenities, and choice and continuity of care.

Figure 1 shows the ReproQ scoring model. The client's responses can be summarized as (a) eight separate domain scores, (b) the personal summary score (covering the four 'personal' domains) and the setting summary score (covering the four 'setting'-related domains), and (c) the total score (covering all eight domains); a higher score implies better performance. Each score can be presented for each of the four reference 
138 periods. The summary scores of clients can be subsequently aggregated by health care

139 provider, organisational unit, or region.

140 Psychometric analyses support the content and construct validity as well as the test-

141 retest reliability of the questionnaire (Scheerhagen et al. 2015b; Scheerhagen et al.

142 2016). For the remainder of this paper, we will only present the results of the $2^{\text {nd }}$ half of

143 pregnancy, as the ratings of the $1^{\text {st }}$ and $2^{\text {nd }}$ half of the pregnancy are highly associated

144 (ICC=0.83) (Scheerhagen et al. 2018).

145

146 ReproQ data collection

147 The ReproQ data were collected from August 2013 to July 2015 . The data were

148 collected digitally from clients of three large maternity care organizations (that deliver

149 postnatal care at home from childbirth onwards during 7-10 days), from several

150 regionally conducted observational studies that used ReproQ client experiences as

secondary outcome measure, and from clients of 10 perinatal units interested in quality

152 improvement. A total of 60 of 85 Dutch perinatal units participated. Further details have

153 been reported elsewhere (Scheerhagen et al. 2016).

154 There were no exclusion criteria. All women could participate provided that they gave

155 informed consent. Invitations to fill out the antepartum ReproQ were sent around $34^{\text {th }}$

156 weeks' gestational age. The postpartum ReproQ was sent 6 weeks after the expected 
157 date of childbirth. Non-responding clients received a reminder two weeks after the initial

158 invitation. The study protocol and procedures were approved by the Medical Research

159 Ethics Review Board, Erasmus Medical Center, Rotterdam, the Netherlands (MEC-

160 2013-455).

161

162 Unit of analysis: Perinatal unit

163 The perinatal unit was the unit of analysis. At the time the study was conducted, each

164 perinatal unit contained one and only one hospital. Consequently, clients were allocated

165 to the hospital's perinatal unit in case of a hospital birth. In case of an out-of-hospital

166 birth, clients were allocated to the hospital (and perinatal unit) that was closest to the

167 client's home address. Descriptive characteristics of perinatal units were obtained from

168 various public sources (Posthumus et al. 2015); (STZ Foundation 2014); (PRN

169 Foundation 2013).

170

171 Excluded data

172 Excluded from analysis were: (1) ReproQ data of clients who could not be allocated to

173 one perinatal unit (72 clients, $0.7 \%$ ); (2) ReproQ data with $>50 \%$ missing answers in two

174 or more ReproQ domains; and (3) data of perinatal units who included less than 50

175 clients. 
177 Analytical framework: multilevel analysis

178 Crude differences in summary ReproQ scores across perinatal units, the dependent variable, may originate from three sources: 1) 'actual' differences across perinatal units,

2) differences in client characteristics across perinatal units, and 3) residual variance.

181 Given the hierarchical data structure (perinatal units, and clients within perinatal units), existing differences in client characteristics across perinatal units may obscure the estimation of the 'actual' difference across units. In that case, multi-level analysis is the appropriate method to decompose total data variance into variance attributable to perinatal units (source 1) and variance attributable to other sources (sources 2+3), in particular variance related to client characteristics (Twisk 2014). Estimation of the 'actual' difference between perinatal units (source 1) requires the domain and summary scores to be corrected for the other variance components (typically client characteristics), as systematic client heterogeneity may bias and limit the comparison of perinatal units; i.e. case mix correction. The Technical Supplement shows further details on the multilevel analysis and software used.

194 For a fair ranking of units, we explored the need for case mix correction. We 
195

196

197

198

199

200

201

202

203

204

\section{1}

distinguished the following groups of potential casemix variables: 1) socio-demographic

variables; 2) process of care variables; 3) interventions; and 4) perceived (client-

reported) outcomes. The variables in the casemix correction were limited to variables

that cannot be modified by perinatal units and healthcare providers, i.e. socio-

demographic variables and perceived (patient reported) outcomes.

To explore the need for casemix correction, we analyzed two models: (1) an 'empty'

model with the ReproQ domain or summary scores as dependent variable, and a

random intercept for each perinatal unit; (2) an adjusted model, with the ReproQ domain

or summary score as dependent variable, a random intercept for each perinatal unit, and client characteristics included as explanatory variables (Bos et al. 2015; Stubbe et

al. 2007). Of the available client characteristics (age, educational level, ethnicity, parity,

and client-reported health) only age, educational level, and client-reported health

contributed significantly $(p<0.05)$ to all domain and summary scores, and were therefore

included in the casemix correction in all analyses. We also tested for random slopes,

but none of these were significant and therefore remain unreported.

\section{Discriminative ability: Two approaches}

We used two complementary approaches to determine discriminative power. 
Approach 1. Multilevel testing of the deviation of unit means from overall (grand) mean

216 Multi-level analyses were used to examine to what degree ReproQ is able to identify

217 units that significantly perform above and below average (averaged over perinatal

218 units), producing three parts of information:

219 1) Estimated variance components and ICCs. The ICC of interest is the ratio of the variance in perinatal units and the variance in client's characteristics in that unit

221 (Streiner et al. 2014). An ICC close to zero implies that the client's experience is

222 unrelated to the perinatal unit in which one receives care; an ICC close to one means

223 that the perinatal unit is of decisive importance. Best and poor performing units are

224 identified by the deviation of the $95 \% \mathrm{Cl}$ of each individual perinatal unit from the grand

225 mean of all perinatal units.

226 2) Estimated G-coefficients, which represent the proportion of variance in the unit-level

227 mean scores attributable to 'actual' variation among perinatal units. A G-coefficient of 228 one implies that all variance in domain and summary scores across perinatal units is

229 attributed to the perinatal unit, and no variance can be attributed to other sources.

3) Estimation of the minimal number of clients needed per perinatal unit to achieve

231 sufficient reliability (D-Study), in our study defined as 0.80 . Small numbers of clients and 
232 large heterogeneity in client experiences produce wide confidence intervals, but only the

233 numbers of clients can be influenced.

234 The conventional mode of presentation is the so-called caterpillar-plot; see Figure 2.

235

236 [Figure 2]

237

238

Approach 2. Relevant deviation based on MID

This approach judges ReproQ's discriminative power on the basis of the ability to demonstrate a relevant difference in domain or summary scores at the perinatal unit level, defined as a difference score beyond the so-called minimally important difference (MID). Underperforming units are identified as those units with domain or summary scores below a certain threshold that equals the mean score domain or summary score of the $10 \%$ best performing units minus the MID. We previously determined, at the individual level, the minimally important difference (MID) for both the summary and domain scores of the childbirth period (Scheerhagen et al. 2016). For the study reported

247 here, we derived MIDs for the late pregnancy and postnatal reference periods in a 248 similar way. Note that a difference of 1.0 unit of MID at the perinatal unit level reflects a large difference: it means that all clients cared for in that unit, on average differ 1.0 MID 250 from a reference value, either being much better (best practice) or much worse (poor practice). We also presented results for a more conservative 0.5 MID. 
Profiling underperforming units

254 Assuming sufficient discriminatory power, we explored ReproQ's ability to profile underperforming perinatal units, once these units have been identified. For that purpose, we compared the domain scores (reference period: childbirth) of the statistically best performing perinatal unit with the significantly underperforming perinatal units. Subsequently, once the underperforming domains have been identified, profiling can be applied to the items of the domains. A detailed comparison of the items may provide clues to the precise activity (or activities) where underperforming units should improve. We illustrate this stepwise approach of underperformance to guide quality improvement using the Communication-domain.

\section{Results}

We invited 27,487 pregnant women to participate in the antepartum ReproQ (response:

$8,567(31 \%))$ and 37,230 women who recently had given birth to respond to the postpartum ReproQ (response: 12,477 (39\%)). Excluded from analysis were 1,419 pregnant women and 1,751 women who recently had given birth, for having $>50 \%$ missing answers. Additionally, we excluded 761 pregnant women and 1,080 women who recently had given birth, for whom the perinatal unit code was missing or being a

272 perinatal unit with less than 50 responses. 
274 [Table 1]

275

276 Table 1 shows the characteristics of participating clients and perinatal units. Differences

277 between the antepartum and postpartum client and unit characteristics were minimal,

278 and about representative for the national pregnancy population.

279

280

[Table 2]

281

282

Table 2 presents the results of the multi-level analysis of the corrected model which

includes perinatal unit as random effect and the client characteristics age, educational

level and client-reported health as case mix correction variables. Each row represents a

separate analysis for the experience measure shown (total score, domain score) in

each of the three periods (pregnancy, childbirth, postnatally). Columns \#2-4 provide the

estimated ICCs, or the ratio of variance assigned to perinatal units (column \#2) and

variance assigned to client characteristics (column \#3). For example, the first row shows

the results for the antepartum total ReproQ score. It shows that little variance on the

client level can be assigned to the perinatal units in general (0.0001), and somewhat

more to the variance of client characteristics (0.064). The ICC is 0.011 (column \#4), 
292 indicating that the client's experience is to a limited extent related to the perinatal unit in

293 which one received care. Column \#5 shows that the G-coefficients (or proportion of

294 variance in the mean scores of perinatal units that can be attributed to the 'actual'

295 variation among perinatal units; the higher the better) of the total score during

296 pregnancy is 0.63 . Finally, the $6^{\text {th }}$ column shows that at least 272 respondents per unit

297 are needed to achieve a reliability (G-coefficient) of 0.80 .

298 As Table 2 shows, all ICCs of the three summary scores for all three reference periods

were lower than 0.03 . Moreover, the ICCs of the case mix corrected models range from

0.002 (the domains dignity, confidentiality and choice \& continuity during childbirth) to

0.125 (communication during childbirth). Moreover, the ICCs for the individual domains

showed more variability than the summary scores, with Communication showing the

highest ICC.

The G-coefficients of the summary scores ranged from 0.45 (personal score during pregnancy) to 0.75 (setting score during postnatal period). The G-coefficients of the domain scores ranged between 0.19 (dignity during childbirth) to 0.93 and 0.96

(communication during childbirth and postnatal period). For the antepartum period,

prompt attention (0.74) and basic amenities (0.70), both part of the setting score, were the domains with highest G-coefficients. 
311 (Communication during Childbirth) to 1,910 (Dignity during Childbirth). The total scores

312 of the ReproQ would achieve excellent reliability (G-coefficient of 0.80 ) when all

313 perinatal units would have included 272 (antepartum), 432 (childbirth), and 258

314 (postnatal period) valid responses.

315

316 Figure 2 shows the caterpillar-plot for the communication domain during childbirth.

317 Depicted are the corrected means (and $95 \%$ Cls) of all 55 perinatal units, which allows

318 for comparison with the grand mean of all perinatal units. The varying $\mathrm{Cl}$ widths point to

319 heterogeneity (after case mix correction) and different sample sizes per unit. For example, unit \#22 performs only moderately better, and does so significantly, due to its small dispersion.

[Table 3]

Table 3 presents the discriminative power according to the statistical and relevancebased approaches. Using the total score during pregnancy ( $1^{\text {st }}$ row) discriminative power according to the statistical approach would imply that three perinatal units showed a significantly better total score compared to the grand mean (column \#3), 38 units where about average (column \#4), and one unit showed a below-average score 
330 (column \#5). For the total score, discriminative power using the statistical approach was

331 largest for the postnatal period ( $10 / 55$ units being deviant), followed by childbirth (5/55

332 being deviant) and the antepartum period (4/38 being deviant). Of the summary scores,

333 only the personal score in the antenatal period did not statistically discriminate. Overall,

334 the domains communication and basic amenities during childbirth (with 23/55 and 16/55

335 units, respectively, being deviant) and during the postnatal period ( $23 / 55$ and $15 / 55$,

336 respectively) were the domains that discriminated best, due its high reliability (see Table 337 2).

338

Table 3 columns \#6-9 reveal ReproQ's discriminative power based on the MID. For the

total score during pregnancy ( $1^{\text {st }}$ row), the $10 \%$ best performing units have a mean total

score of 3.80 (reference value). The corresponding MID is 0.11 . Applying this MID of

0.11 implies that seven perinatal units with their $\mathrm{Cl}$ perform below this reference. For the

summary scores, the number of perinatal units that differed more than 1.0 unit of MID

compared to the reference value ranged from seven (both the total and personal scores

in pregnancy) to 29 (setting score in the postnatal period). The domains with most

discriminating power differed for the three reference periods: autonomy and basic

347 amenities during pregnancy, basic amenities and social considerations during childbirth,

348 and communication, social considerations and choice \& continuity during postnatal 
349 period (see column \#8). Applying a conservative 0.5 unit of MID considerably increased

350 the number of units that relevantly deviated from reference for all scores and reference

351 periods.

352

353

354 Figures 3 and 4 illustrate the potential of ReproQ to profile units being selected as poor

355 performer. Figure 3 displays the domain scores of the single best and four worst

356 performing perinatal units, with best and underperformance defined according to the

357 total score during childbirth. Poor performing units showed systematically lower scores

358 for all domains.

359

$360 \quad$ [Figure 3]

361

362 Figure 4 displays the profiles of the four underperforming perinatal units by

363 disaggregating the Communication domain score into its item scores. The low

364 Communication domain score was predominantly associated with one specific item:

365 'giving consistent advice'.

366

$367 \quad$ [Figure 4]

368

369 


\section{Discussion}

371 ReproQ, a validated instrument for measuring service quality in maternity care, has the

372 ability to discriminate well across perinatal units using two complementary approaches;

373 a multilevel significance-based approach and a relevance-based analysis (MID). It did

374 so successfully despite four conditions that could limit its discriminative performance: a

375 predominantly healthy and relatively homogenous population, standardized care

376 procedures, a naturalistic study design, and the use of aggregated means. Using the

377 total score during childbirth, the significance approach identified four underperforming

378 units, whereas the MID-based approach identified 10 underperforming units using a

$37910 \%$ best-practice norm and 1.0 MID as cut-off. Once the underperforming units were

380 identified, ReproQ domain and item scores provided useful disaggregated information

381 for quality improvement.

A study strength is that sample size was large and data were collected in routine practice, covering about $2 / 3$ s of perinatal units. Clients and practices covered the full range of relevant characteristics, adding to generalizability. Secondly, the significancebased and relevance-based approaches yielded consistent results, with the latter approach displaying considerably more sensitivity. The significance-based approach 
389 tests if 'unit' has a significant impact on outcomes. Significance relies on the number of

390 respondents, case mix correction, and details of the multilevel analysis, with the intrinsic

391 risk that homogeneity of clients and units can lead to significant results, even when

392 these differences are not meaningful. The reverse is more common: client heterogeneity

393 within units and measurement error can obscure relevant unit differences. The

394 relevance-based approach inevitably relies on the chosen reference (i.e. $10 \%$ best

395 units) and magnitude of the MID (i.e. 0.5 or 1.0 MID as threshold). One should be aware

396 that all our choices in both approaches were rather conservative, and that an average

397 difference of 1.0 MID at the unit level expresses a rather extreme difference. Other

398 studies on client experiences only explored the discriminative power in statistical terms

399 (Bos et al. 2015; De Boer et al. 2011; Krol et al. 2015; Stubbe et al. 2007). We believe

400 the MID-based approach is a necessary complement to the significance-based

401 approach.

402

A third strength is that we avoided overfitting and overcorrection by limiting case mix

403 correction to predefined candidate factors with an accepted established effect (Sixma et

404 al. 2008).

405

406 Two limitations merit discussion. Firstly, while the respondents were largely

407 representative for the pregnancy population, non-Western women were somewhat 
408 underrepresented (8\% vs. national average 14\% (PRN Foundation 2013)). Since non-

409 Western women tend to report more negative experiences than Western women

410 (Scheerhagen et al. 2015a), increased participation of non-Western women would

411 probably lower the average summary scores but not affect the ranking of perinatal units,

412 as our case mix analysis did not reveal a significant role of ethnicity. Regarding age,

413 women younger than 24 years were slightly underrepresented ( $6 \%$ vs $11 \%)$, as were

414 women with a low educational level (6-8\% vs. $18 \%)$. These gaps appear modest in

415 quantitative terms. More important, a study we conducted recently on the determinants

416 of experience scores showed that maternal age and educational level have no

417 significant impact on the level of experience scores (Scheerhagen et al. 2019).

418 Reference data on client-reported health status are absent.

420 Secondly, we did not include the individual professional as additional level in the

421 analysis. One may assume an effect of individual professional's behaviour on the

personal domains rather than the setting domains, and its impact is probably larger than

423 the variation across units (Krol et al. 2015; Roberts et al. 2014). While the primary focus

424 of quality improvement is the unit, one should be aware of the professional's role in

425 quality improvement cycles. Inclusion of the professional in the analysis would require a 
426 highly detailed, perhaps unfeasible, registration of all caregivers involved the care

427 process.

428

429 Three technical remarks can be made. First, a study of performance at the domain level

430 requires about 450 respondents per unit, which is considerably higher than the

431 minimum of 10 respondents per unit adopted in similar studies (Bos et al. 2015; Stubbe

432 et al. 2007). The view that 10 respondents are representative for a unit's performance is

433 highly questionable, given the variability in respondent characteristics, in experiences

434 within a unit, and in the care provided. A sample size of 450 clients is well below the

435 average unit size of 2,000 clients, implying that a sampling approach instead of all-client

436 measurement should suffice.

437 Secondly, although the estimated ICCs appeared low, they are comparable to the ICCs

438 of other accepted client experience questionnaires (Bos et al. 2015; De Boer et al.

439 2011; Krol et al. 2015; Stubbe et al. 2007). ICCs are low because the denominator

440 essentially is the number of client questionnaires. The impact of each unit on each

441 individual questionnaire is small. Small effects at the client level may build up as large

442 effects at the unit level.

444 Finally, the systematic effect of perinatal unit was stronger during childbirth and 
445 postnatally than antenatally. The likely explanation is that antenatal care is highly

446 standardized in terms of procedures and professionals involved whereas different

447 processes, adverse events and outcomes do emerge during childbirth and postnatally,

448 where unit quality is challenged. This observation emphasizes that the very assumption

449 that quality differs across units may not be true when care is highly standardized. In that

450 case, differences across units truly are small, causing low ICCs and lack of

451 discrimination. We believe that favorable antenatal performance should be interpreted

452 primarily as uniform performance rather than good performance (Scheerhagen et al.

453 2015b; Scheerhagen et al. 2016). This phenomenon has been described with other

454 client experience questionnaires as well (Peterson et al. 2005; Redshaw \& Heikkila

455 2010b; Smith 2011).

456

457 Once underperforming units have been identified, profiling of its items (which are described in terms of activities) may guide interventions to improve service quality.

459 Qualitative interviews and client involvement may further support the interpretation of domain and item scores. Also research into the varying performance of client

461 subgroups, e.g. deprived clients, is recommended (Department of Health 2010); Ellis

462 (2006); (Ettorchi-Tardy et al. 2012; Hitzert et al. 2016; Kay 2007). After identifying areas 463 that need improvement, experts could be consulted to inform on the causes of 
464 suboptimal ReproQ domain and item scores and recommend actions for improvement.

465 Here, both clients and health care professionals can take up the role of experts

466 (Groenen et al. 2017). Another strategy is to derive recommendations for quality

467 improvement from multidisciplinary meetings or audits, a strategy occasionally used in

468 maternity care (Alderliesten et al. 2008; Eskes et al. 2014; Kurinczuk et al. 2014;

469 Mancey-Jones \& Brugha 1997).

470

471 For the future, we recommend using ReproQ in maternity care to measure clients'

472 experiences with care and using the results to guide the improvement of the

473 performance in maternity care by means of profiling. Qualitative interview, client

474 involvement and audits may further support this process. This may fit well in outcome-

475 based strategies like those initiated by ICHOM (ICHOM 2016), that includes both

476 medical outcomes and quality of care.

477

478 Conclusion

479 ReproQ, a valid and efficient measure of client experiences in maternity care, has the

480 ability to discriminate well across perinatal units, and is suitable for benchmarking under

481 routine conditions. 
We are grateful to Koen Ilja Nijenhuijs for his assistance with the caterpillar plots.

\section{References}

Alderliesten ME, Stronks K, Bonsel GJ, Smit BJ, van Campen MM, van Lith JM, and Bleker OP. 2008. Design and evaluation of a regional perinatal audit. Eur J Obstet Gynecol Reprod Biol 137:141145. 10.1016/j.ejogrb.2007.06.002

Bos N, Sturms LM, Stellato RK, Schrijvers AJ, and van Stel HF. 2015. The Consumer Quality Index in an accident and emergency department: internal consistency, validity and discriminative capacity. Health Expect 18:1426-1438.

Campbell SM, Roland MO, and Buetow SA. 2000. Defining quality of care. Soc Sci Med 51:1611-1625.

Collins-Fulea C, Mohr JJ, and Tillett J. 2005. Improving midwifery practice: the American College of Nurse-Midwives' benchmarking project. $J$ Midwifery Womens Health 50:461-471.

De Boer D, Delnoij D, and Rademakers J. 2011. The discriminative power of patient experience surveys. BMC Health Serv Res 11:332.

De Silva A. 2000. A framework for measuring responsiveness. Global Programme on Evidence for Health Policy Discussion Paper 32.

Department of Health. 2010. Essence of Care 2010. Crown: The Stationery Office.

Dzakpasu S, Kaczorowski J, Chalmers B, Heaman M, Duggan J, and Neusy E. 2008. The Canadian maternity experiences survey: design and methods. J Obstet Gynaecol Can 30:207-216.

Ellis J. 2006. All inclusive benchmarking. J Nurs Manag 14:377-383.

Eskes M, Waelput AJ, Erwich JJ, Brouwers HA, Ravelli AC, Achterberg PW, Merkus HJ, and Bruinse HW. 2014. Term perinatal mortality audit in the Netherlands 2010-2012: a population-based cohort study. BMJ Open 4:e005652. 10.1136/bmjopen-2014-005652

Ettorchi-Tardy A, Levif M, and Michel P. 2012. Benchmarking: A method for continuous quality improvement in health. Healthcare Policy 7:e101-e119.

Gostin L. 2002. The domains of health responsiveness: a human rights assessment.

Groenen CJM, Van Duijnhoven NTL, Kremer JA, Scheerhagen M, Vandenbussche FPHA, and Faber MJ. 2017. Shared agenda making for quality improvement; towards more synergy in maternity care. European journal of obstetrics gynecology and reproductive biology 219:15-19.

Hay H. 2010. A report on the development of the questionnaire for the 2010 maternity survey. Available at http://www.nhssurveys.org/surveys/483

Hitzert MF, Hermus MAA, Scheerhagen M, Boesveld IC, Wiegers T, Akker-van Marle ME, Van Dommelen P, Van der Pal-de Bruin KM, and De graaf JP. 2016. Experiences of women who planned birth in a birth centre compared to alternative planned places of birth. Results of a Dutch birth centre study. Midwifery 40:70-78.

ICHOM. 2016. Pregnancy and childbirth. Available at http://www.ichom.org/medicalconditions/pregnancy-and-childbirth/ (accessed july, 1st 2018).

Kay JFL. 2007. Health care benchmarking. Hong Kong Medical Diary 12:22-27.

Krol MW, De Boer D, Sixma H, Van Der Hoek L, Rademakers JJ, and Delnoij DM. 2015. Patient experiences of inpatient hospital care: a department matter and a hospital matter. Int J Qual Health Care 27:17-25.

Kurinczuk JJ, Draper ES, Field DJ, Bevan C, Brocklehurst P, Gray R, Kenyon S, Manktelow BN, Neilson JP, Redshaw M, Scott J, Shakespeare J, Smith LK, and Knight M. 2014. Experiences with maternal and perinatal death reviews in the UK--the MBRRACE-UK programme. Bjog 121 Suppl 4:41-46. 10.1111/1471-0528.12820

Peer) reviewing PDF | (2019:02:34802:1:2:NEW 14 Jul 2019) 
528

529

530

531

532

533

534

535

536

537

538

539

540

541

542

543

544

545

546

547

548

549

550

551

552

553

554

555

556

557

558

559

560

561

562

563

564

565

566

567

568

569

570

571

572

573

574

575

576

577

578

579

580

581

582

583

Mancey-Jones M, and Brugha RF. 1997. Using perinatal audit to promote change: a review. Health Policy Plan 12:183-192.

Mohangoo AD, Buitendijk SE, Szamotulska K, Chalmers J, Irgens LM, Bolumar F, Nijhuis JG, Zeitlin J, and Euro-Peristat Scientific C. 2011. Gestational age patterns of fetal and neonatal mortality in Europe: results from the Euro-Peristat project. PLoS One 6:e24727.

Peterson WE, Charles C, DiCenso A, and Sword W. 2005. The Newcastle Satisfaction with Nursing Scales: a valid measure of maternal satisfaction with inpatient postpartum nursing care. J Adv Nurs 52:672-681. JAN3634 [pii]

10.1111/j.1365-2648.2005.03634.x

Posthumus AG, Scholmerich VL, Steegers EA, Kawachi I, and Denktas S. 2015. The association of ethnic minority density with late entry into antenatal care in the Netherlands. PLoS One 10:e0122720.

PRN Foundation. 2013. Netherlands Perinatal Registry. 2004-2013

Redshaw M, and Heikkila K. 2010a. Delivered with care: A national survey of women's experience of maternity care. Available at https://www.npeu.ox.ac.uk/delivered-with-care.

Redshaw M, and Heikkila K. 2010b. Delivered with care: a national survey of women's experience of maternity care. Oxford, United Kingdom: National Perintal Epidemiology Unit.

Roberts MJ, Campbell JL, Abel GA, Davey AF, Elmore NL, Maramba I, Carter M, Elliott MN, Roland MO, and Burt JA. 2014. Understanding high and low patient experience scores in primary care: analysis of patients' survey data for general practices and individual doctors. Bmj 349:g6034.

Scheerhagen M, Birnie E, Franx A, van Stel HF, and Bonsel GJ. 2018. Measuring clients' experiences with antenatal care before or after childbirth: it matters. PeerJ e5851.

Scheerhagen M, Brinie E, Franx A, van Stel HF, and Bonsel GJ. 2019. What determines women's birth experiences? Applications for a benchmark. Rotterdam: Erasmus University Rotterdam.

Scheerhagen M, Van Stel HF, Birnie E, Franx A, and Bonsel GJ. 2015a. Het discriminerend vermogen van de ReproQuestionnaire (the discriminative power of the ReproQuestionnaire). Rotterdam, The Netherlands: Erasmus Medical Center.

Scheerhagen M, Van Stel HF, Birnie E, Franx A, and Bonsel GJ. 2015b. Measuring Client Experiences in Maternity Care under Change: Development of a Questionnaire Based on the WHO Responsiveness Model. PLoS One 10:e0117031. 10.1371/journal.pone.0117031

PONE-D-14-18958 [pii]

Scheerhagen M, Van Stel HF, Tholhuijsen DJ, Birnie E, Franx A, and Bonsel GJ. 2016. Applicability of the ReproQ client experiences questionnaire for quality improvement in maternity care. PeerJ 4:e2092. 10.7717/peerj.2092

Sixma H, Hendriks M, De Boer D, and Delnoij D. 2008. Manual CQI development: guidelines and regulations for the development of a CQI measurement [Handboek CQI ontwikkeling: richtlijnen en voorschriften voor de ontwikkeling van een CQI meetinstrument]. Available at http://www.nivel.nl/sites/default/files/bestanden/Handboek-CQI-Ontwikkeling.pdf.

Smith LF. 2011. Postnatal care: development of a psychometric multidimensional satisfaction questionnaire (the WOMBPNSQ) to assess women's views. Br J Gen Pract 61:e628-637. 10.3399/bjgp11X601334

Streiner DL, Norman GR, and Cairney J. 2014. Health measurement scales. Oxford: University Press.

Stubbe JH, Brouwer W, and Delnoij DM. 2007. Patients' experiences with quality of hospital care: the Consumer Quality Index Cataract Questionnaire. BMC Ophthalmol 7:14.

STZ Foundation. 2014. http://www.stz.nl/1087/over-ons/stz-ziekenhuizen (accessed October, 24th 2015).

Twisk JWR. 2014. Applied Multilevel Analysis: Cambridge University Press.

Valentine NB, Bonsel GJ, and Murray CJ. 2007. Measuring quality of health care from the user's perspective in 41 countries: psychometric properties of WHO's questions on health systems responsiveness. Qual Life Res 16:1107-1125.

Valentine NB, De Silva A, Kawabata K, Darby C, Murray CJ, and Evans BE. 2003. Health system responsiveness: concepts, domains and measurement. In: Murray $\mathrm{CJ}$, and Evans BE, eds. Health systems performance assessment. Geneva: World Health Organization, 573-596.

Van Wagtendonk I, Hoek Vd, and Wiegers T. 2010. Development of a consumer quality index of postnatal care. Available at http://www.zorginstituutnederland.nl/kwaliteit/toetsingskader+en+register/cq-index/cqivragenlijsten - CQIKraamzorg

Peer] reviewing PDF | (2019:02:34802:1:2:NEW 14 Jul 2019) 
584 Wiegers TA, Keirse MJ, Berghs GA, and van der Zee J. 1996. An approach to measuring quality of 585 midwifery care. J Clin Epidemiol 49:319-325.

Zeitlin J, Mohangoo AD, Delnord M, Cuttini M, and Committee E-PS. 2013a. The second European Perinatal Health Report: documenting changes over 6 years in the health of mothers and babies in Europe. J Epidemiol Community Health 67:983-985.

Zeitlin J, Szamotulska K, Drewniak N, Mohangoo AD, Chalmers J, Sakkeus L, Irgens L, Gatt M, Gissler M, Blondel B, and Euro-Peristat Preterm Study G. 2013b. Preterm birth time trends in Europe: a study of 19 countries. Bjog 120:1356-1365.

592 
Figure 1

ReproQ's scoring model 


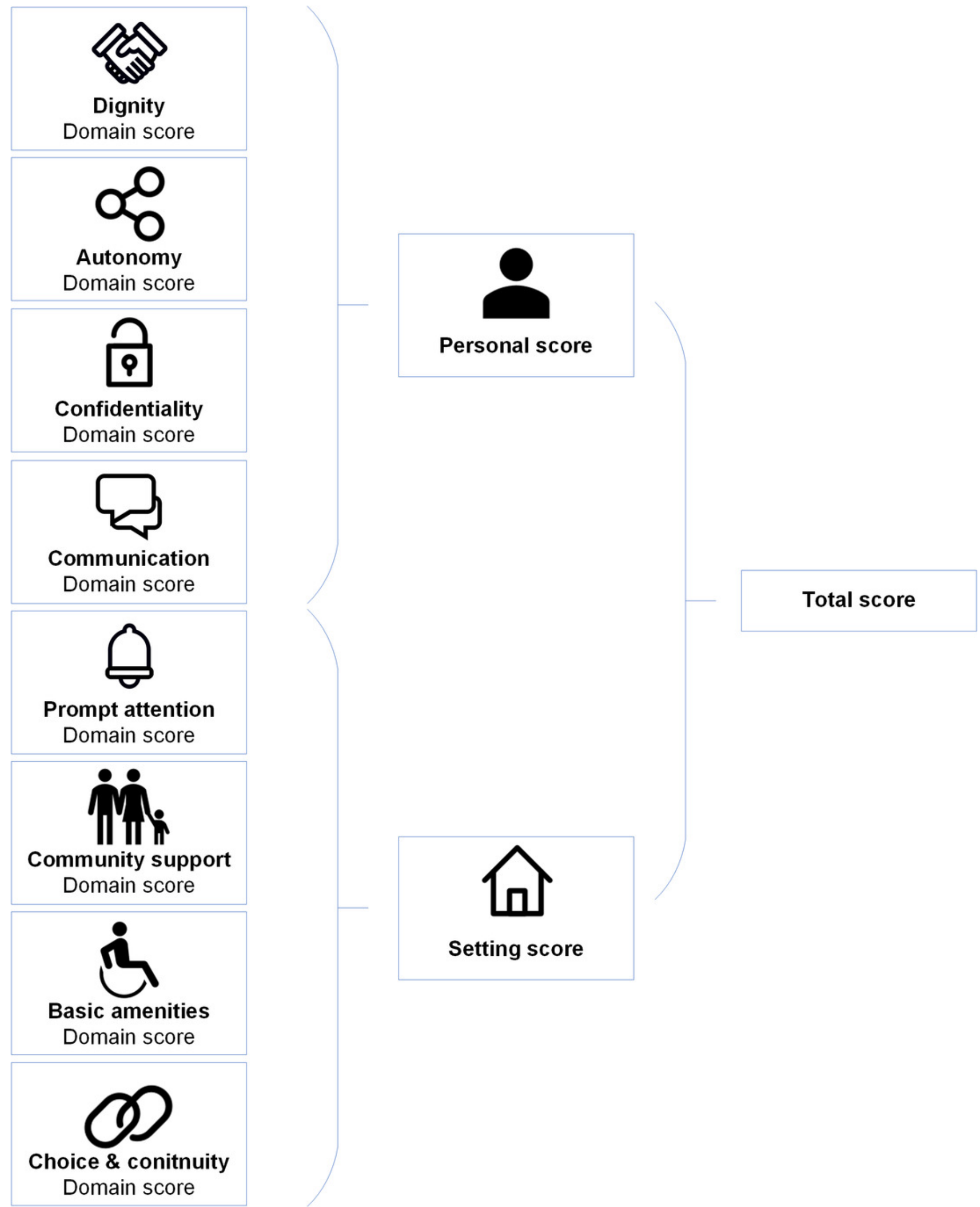


Figure 2

Caterpillar-plot: Ranking of perinatal units for the domain Communication during childbirth ( $\left.\mathrm{N}_{\mathrm{Pu}}=55\right)$.

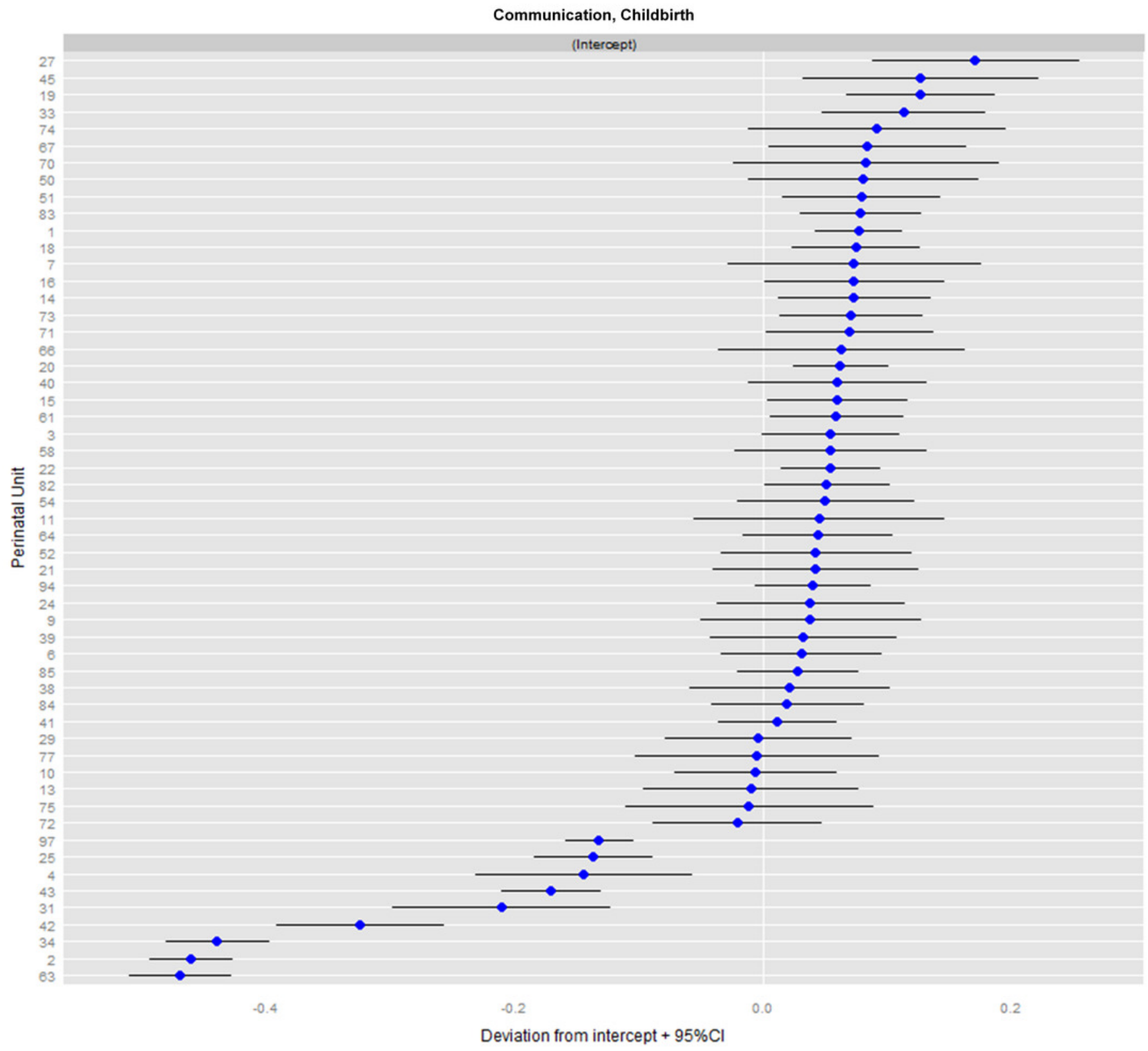


Figure 3

ReproQ domain scores of the single best practice and the four worst performing units during childbirth.

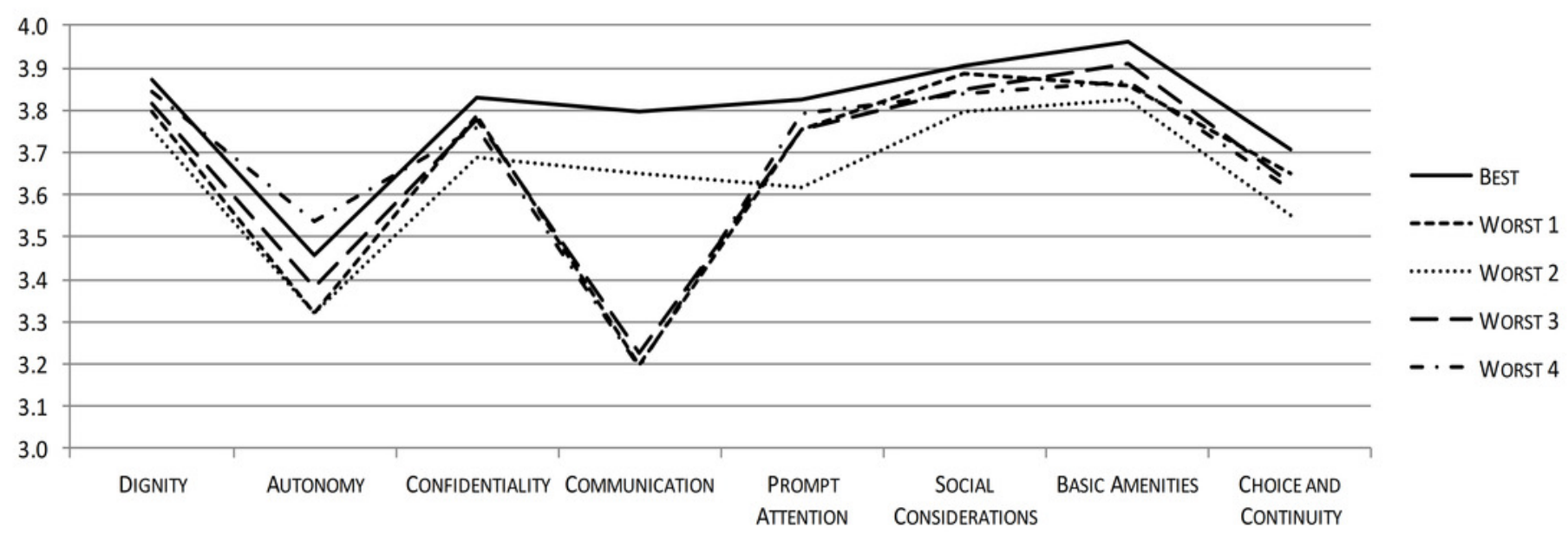




\section{Figure 4}

\section{ReproQ item scores of the Communication-domain of the single best practice and the four worst performing units during childbirth.}

\begin{abstract}
GIVING CONSISTENT ADVICE (BEST) GIVING CONSISTENT ADVICE (WORST 1) GIVING CONSISTENT ADVICE (WORST 2) GIVING CONSISTENT ADVICE (WORST 3) GIVING CONSISTENT ADVICE (WORST 4)
\end{abstract}

ANSWERING ALL OF THE CLIENT'S QUESTIONS (BEST) ANSWERING ALL OF THE CLIENT'S QUESTIONS (WORST 1) ANSWERING ALL OF THE CLIENT'S QUESTIONS (WORST 2) ANSWERING ALL OF THE CLIENT'S QUESTIONS (WORST 3) ANSWERING ALL OF THE CLIENT'S QUESTIONS (WORST 4)

KEEPING THE CLIENT INFORMED (BEST) KEEPING THE CLIENT INFORMED (WORST 1) KEEPING THE CLIENT INFORMED (WORST 2) KEEPING THE CLIENT INFORMED (WORST 3) KEEPING THE CLIENT INFORMED (WORST 4)

GIVING UNDERSTANDABLE EXPLANATIONS (BEST) GIVING UNDERSTANDABLE EXPLANATIONS (WORST 1 ) GIVING UNDERSTANDABLE EXPLANATIONS (WORST 2) GIVING UNDERSTANDABLE EXPLANATIONS (WORST 3) GIVING UNDERSTANDABLE EXPLANATIONS (WORST 4)

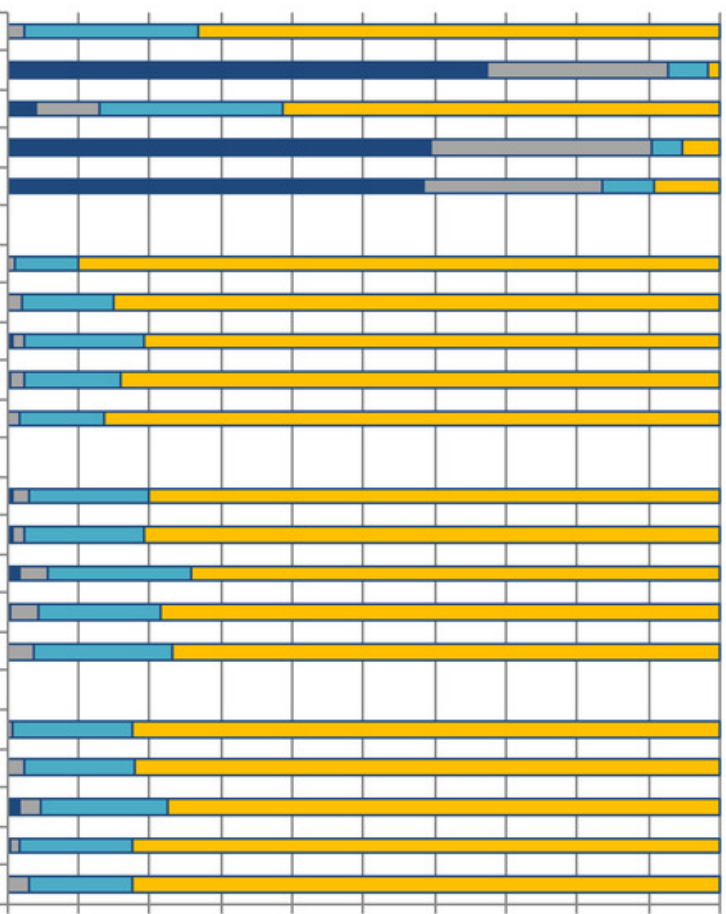

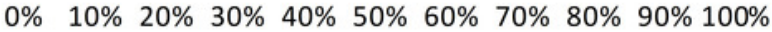

nEVER

口 SOMETIMES

$\square$ MOST OF THE TIME

$\square$ ALWAYS 


\section{Table $\mathbf{1}$ (on next page)}

Characteristics of the participating women $\left(n_{\text {antepartum }}=6,387 ; n_{\text {postpartum }}=9,646\right)$ and perinatal units ( $n_{\text {antepartum }}=42 ; n_{\text {postpartum }}=55$ ).

(A) Mean age was 30.1 years (SD=4.5). (B) Educational level; low 0-6 years; middle 6-12 years; high >12 years. (C) Mean number of respondents per perinatal unit was 152 (range: 54-363) for the antenatal period, and 175 (range: 50-812) for the postnatal period. 
1

\begin{tabular}{|c|c|c|c|c|}
\hline & $\begin{array}{l}\text { Antapartum } \\
\text { questionnaire } \\
\mathrm{N}\end{array}$ & $\%$ & $\begin{array}{l}\text { Postpartum } \\
\text { questionnaire } \\
\mathrm{N}\end{array}$ & $\%$ \\
\hline \multicolumn{5}{|l|}{ Clients } \\
\hline \multicolumn{5}{|l|}{ Age $(A)$} \\
\hline$\leq 24$ & 385 & 6 & 500 & 5 \\
\hline $25-29$ & 2,018 & 32 & 2,730 & 29 \\
\hline $30-34$ & 2,600 & 42 & 4,084 & 43 \\
\hline$\geq 35$ & 1,263 & 20 & 2,197 & 23 \\
\hline \multicolumn{5}{|l|}{ Ethnic background } \\
\hline Western & 5,735 & 92 & 8,711 & 93 \\
\hline Non-Western & 478 & 8 & 696 & 7 \\
\hline \multicolumn{5}{|l|}{ Educational level (B) } \\
\hline Low & 399 & 6 & 754 & 8 \\
\hline Middle & 2,026 & 33 & 3,280 & 35 \\
\hline High & 3,783 & 61 & 5,356 & 57 \\
\hline \multicolumn{5}{|l|}{ Marital status } \\
\hline Married/living together & 5,974 & 96 & 9,052 & 96 \\
\hline $\begin{array}{l}\text { Not living together or no } \\
\text { relationship }\end{array}$ & 226 & 4 & 339 & 4 \\
\hline \multicolumn{5}{|l|}{ Parity } \\
\hline Primiparous & 3,210 & 50 & 4,872 & 51 \\
\hline Multiparous & 3,153 & 50 & 4,735 & 49 \\
\hline \multicolumn{5}{|l|}{ Health status } \\
\hline Poor / moderate & 300 & 5 & 332 & 4 \\
\hline Good & 2,173 & 36 & 3,153 & 33 \\
\hline Very good & 2,390 & 39 & 3,684 & 38 \\
\hline Excellent & 1,244 & 20 & 2,428 & 25 \\
\hline
\end{tabular}

\section{Perinatal units}

Number of respondents (C) 50-99

16

$38 \quad 19$

35

100-149

150-199

$\geq 200$

10

24

14

25

6

14

13

10

24

27

Urbanization

Urban - 4 largest cities

Urban - 10 largest cities,

10

24

5

43

23

93

7

7

except no. 1-4

Rural

6

14

14

25

26

62

35

64

Hopsital type

University hospital

Teaching hospital

Peripheral hospital

Hospital size

$<750$ births a year

$\begin{array}{rrrr}5 & 12 & 6 & 11 \\ 17 & 40 & 20 & 36 \\ 20 & 48 & 29 & 53 \\ 5 & 12 & 6 & 11\end{array}$


750-1499 births a year $\geq 1500$ births a year
20

17
48

40
26

23
47

42

2

PeerJ reviewing PDF | (2019:02:34802:1:2:NEW 14 Jul 2019) 


\section{Table 2 (on next page)}

Results of corrected multi-level analysis model and G-study for ReproQ summary scores and domain scores during pregnancy $(n=6,387)$, and childbirth and postnatal period $(n=9,646)$.

(A) Mean valid response per perinatal unit was 116 antenatally and 109 postnatally. 


\begin{tabular}{|c|c|c|c|c|c|}
\hline & $\begin{array}{l}\text { Variance } \\
\text { of } \\
\text { perinatal } \\
\text { units } \\
\end{array}$ & $\begin{array}{l}\text { Variance of } \\
\text { client } \\
\text { characteristics }\end{array}$ & ICC & $\begin{array}{l}\text { G-coefficient } \\
\text { (A) }\end{array}$ & $\begin{array}{l}\text { Number of } \\
\text { respondents } \\
\text { needed for G- } \\
\text { coefficient of } 0.8\end{array}$ \\
\hline \multicolumn{6}{|l|}{ Pregnancy } \\
\hline Total score & 0.001 & 0.064 & 0.011 & 0.63 & 272 \\
\hline Personal score & 0.000 & 0.071 & 0.004 & 0.45 & 580 \\
\hline Setting score & 0.001 & 0.082 & 0.014 & 0.66 & 243 \\
\hline Dignity & 0.000 & 0.073 & 0.004 & 0.38 & 745 \\
\hline Autonomy & 0.001 & 0.190 & 0.006 & 0.45 & 555 \\
\hline Confidentiality & 0.001 & 0.204 & 0.005 & 0.38 & 755 \\
\hline Communication & 0.000 & 0.115 & 0.004 & 0.35 & 875 \\
\hline Prompt attention & 0.002 & 0.104 & 0.021 & 0.74 & 165 \\
\hline Social considerations & 0.001 & 0.154 & 0.004 & 0.35 & 825 \\
\hline Basic amenities & 0.002 & 0.111 & 0.019 & 0.70 & 202 \\
\hline Choice and continuity & 0.001 & 0.271 & 0.003 & 0.42 & 630 \\
\hline \multicolumn{6}{|l|}{ Childbirth } \\
\hline Total score & 0.001 & 0.075 & 0.009 & 0.50 & 432 \\
\hline Personal score & 0.002 & 0.101 & 0.019 & 0.71 & 176 \\
\hline Setting score & 0.001 & 0.072 & 0.008 & 0.49 & 465 \\
\hline Dignity & 0.000 & 0.115 & 0.002 & 0.19 & 1,910 \\
\hline Autonomy & 0.007 & 0.333 & 0.020 & 0.66 & 210 \\
\hline Confidentiality & 0.001 & 0.214 & 0.002 & 0.23 & 1,465 \\
\hline Communication & 0.021 & 0.147 & 0.125 & 0.96 & 18 \\
\hline Prompt attention & 0.001 & 0.123 & 0.009 & 0.46 & 523 \\
\hline Social considerations & 0.000 & 0.105 & 0.003 & 0.23 & 1,480 \\
\hline Basic amenities & 0.002 & 0.080 & 0.023 & 0.73 & 165 \\
\hline Choice and continuity & 0.001 & 0.260 & 0.002 & 0.23 & 1,440 \\
\hline \multicolumn{6}{|l|}{ Postnatal period } \\
\hline Total score & 0.001 & 0.080 & 0.015 & 0.63 & 258 \\
\hline Personal score & 0.001 & 0.100 & 0.012 & 0.58 & 320 \\
\hline Setting score & 0.002 & 0.081 & 0.025 & 0.75 & 145 \\
\hline Dignity & 0.001 & 0.122 & 0.009 & 0.51 & 425 \\
\hline Autonomy & 0.001 & 0.287 & 0.004 & 0.30 & 1,015 \\
\hline Confidentiality & 0.001 & 0.212 & 0.007 & 0.42 & 600 \\
\hline Communication & 0.011 & 0.145 & 0.073 & 0.93 & 35 \\
\hline Prompt attention & 0.002 & 0.096 & 0.018 & 0.66 & 221 \\
\hline Social considerations & 0.001 & 0.126 & 0.008 & 0.51 & 418 \\
\hline Basic amenities & 0.004 & 0.108 & 0.035 & 0.78 & 123 \\
\hline Choice and continuity & 0.003 & 0.268 & 0.013 & 0.64 & 249 \\
\hline
\end{tabular}




\section{Table 3 (on next page)}

Discriminative power of the ReproQ based on statistical power (significance perspective) and the ability to detect 1.0 and 0.5 MID difference (relevance perspective) for all ReproQ outcomes during pregnancy $\left(\mathrm{n}_{\mathrm{pu}}=42\right)$, childbirth and postnatal per

(A) The mean best-practices is the pooled average of the $10 \%$ best performing units. 


\begin{tabular}{|c|c|c|c|c|c|c|c|c|}
\hline & \multirow[t]{2}{*}{$\begin{array}{l}\text { Overall } \\
\text { mean }\end{array}$} & \multicolumn{3}{|c|}{$\begin{array}{c}\text { Discriminative power based on } \\
\text { statistics }\end{array}$} & \multicolumn{4}{|c|}{ Discriminative power based on relevance } \\
\hline & & $\begin{array}{l}\text { Best- } \\
\text { practices }\end{array}$ & Average & $\begin{array}{l}\text { Under } \\
\text { performers }\end{array}$ & $\begin{array}{l}\text { Mean best } \\
\text { practices }(\mathrm{A}) \\
(\Delta \mathrm{P} 90-\mathrm{P} 100)\end{array}$ & MID & $\begin{array}{l}\text { Under } \\
\text { performers } \\
\text { ( } \triangle \mathrm{P} 90 \text { 1MID) }\end{array}$ & $\begin{array}{l}\text { Under } \\
\text { performers } \\
\text { ( } \triangle \mathrm{P} 900.5 \mathrm{MID})\end{array}$ \\
\hline \multicolumn{9}{|l|}{ Pregnancy } \\
\hline Total score & 3.73 & 3 & 38 & 1 & 3.80 & 0.11 & 7 & 22 \\
\hline Personal score & 3.75 & 0 & 42 & 0 & 3.81 & 0.09 & 7 & 28 \\
\hline Setting score & 3.72 & 3 & 35 & 4 & 3.79 & 0.12 & 9 & 23 \\
\hline Dignity & 3.87 & 0 & 42 & 0 & 3.91 & 0.07 & 10 & 25 \\
\hline Autonomy & 3.65 & 1 & 40 & 1 & 3.75 & 0.11 & 21 & 34 \\
\hline Confidentiality & 3.73 & 0 & 41 & 1 & 3.81 & 0.09 & 12 & 36 \\
\hline Communication & 3.75 & 0 & 42 & 0 & 3.80 & 0.11 & 6 & 21 \\
\hline Prompt attention & 3.67 & 5 & 32 & 5 & 3.77 & 0.11 & 19 & 30 \\
\hline Social considerations & 3.76 & 0 & 42 & 0 & 3.84 & 0.09 & 16 & 30 \\
\hline Basic amenities & 3.81 & 4 & 34 & 4 & 3.89 & 0.08 & 22 & 29 \\
\hline $\begin{array}{l}\text { Choice and continuity } \\
\text { Childbirth }\end{array}$ & 3.63 & 0 & 42 & 0 & 3.74 & 0.19 & 7 & 26 \\
\hline Total score & 3.73 & 1 & 50 & 4 & 3.80 & 0.10 & 10 & 40 \\
\hline Personal score & 3.66 & 5 & 46 & 4 & 3.75 & 0.11 & 13 & 39 \\
\hline Setting score & 3.79 & 4 & 50 & 1 & 3.86 & 0.08 & 21 & 44 \\
\hline Dignity & 3.83 & 1 & 54 & 0 & 3.88 & 0.09 & 9 & 35 \\
\hline Autonomy & 3.44 & 7 & 42 & 6 & 3.61 & 0.17 & 25 & 43 \\
\hline Confidentiality & 3.77 & 0 & 55 & 0 & 3.84 & 0.08 & 32 & 49 \\
\hline Communication & 3.62 & 15 & 32 & 9 & 3.80 & 0.11 & 17 & 44 \\
\hline Prompt attention & 3.77 & 1 & 51 & 3 & 3.84 & 0.10 & 18 & 42 \\
\hline Social considerations & 3.86 & 0 & 55 & 0 & 3.92 & 0.04 & 42 & 50 \\
\hline Basic amenities & 3.88 & 7 & 39 & 9 & 3.94 & 0.05 & 36 & 48 \\
\hline Choice and continuity & 3.66 & 1 & 54 & 0 & 3.77 & 0.13 & 23 & 44 \\
\hline \multicolumn{9}{|l|}{ Postnatal period } \\
\hline Total score & 3.74 & 5 & 45 & 5 & 3.83 & 0.10 & 18 & 45 \\
\hline Personal score & 3.71 & 4 & 47 & 4 & 3.80 & 0.10 & 19 & 46 \\
\hline Setting score & 3.78 & 8 & 39 & 8 & 3.86 & 0.10 & 29 & 42 \\
\hline Dignity & 3.81 & 4 & 48 & 3 & 3.90 & 0.10 & 21 & 45 \\
\hline Autonomy & 3.71 & 3 & 52 & 0 & 3.82 & 0.12 & 31 & 47 \\
\hline Confidentiality & 3.74 & 3 & 50 & 2 & 3.82 & 0.11 & 23 & 41 \\
\hline Communication & 3.59 & 13 & 32 & 10 & 3.75 & 0.09 & 33 & 47 \\
\hline Prompt attention & 3.80 & 6 & 42 & 7 & 3.87 & 0.09 & 21 & 41 \\
\hline Social considerations & 3.81 & 5 & 49 & 1 & 3.89 & 0.07 & 33 & 48 \\
\hline Basic amenities & 3.86 & 7 & 40 & 8 & 3.95 & 0.08 & 27 & 44 \\
\hline Choice and continuity & 3.65 & 5 & 49 & 1 & 3.79 & 0.14 & 34 & 48 \\
\hline
\end{tabular}

